\title{
New approaches to bicyclic vinyl heterocycles from propargylic acetals
}

\author{
Frédéric Le Strat, David C. Harrowven and Jacques Maddaluno
}

\section{Supporting Information}

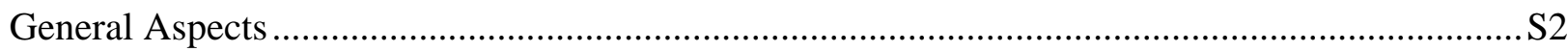

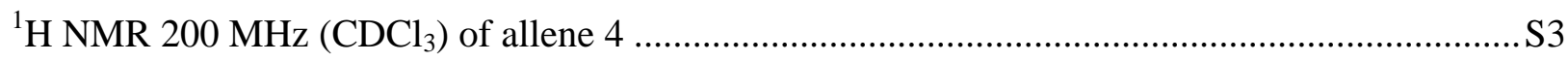

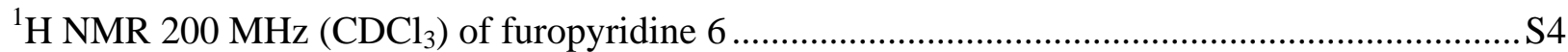

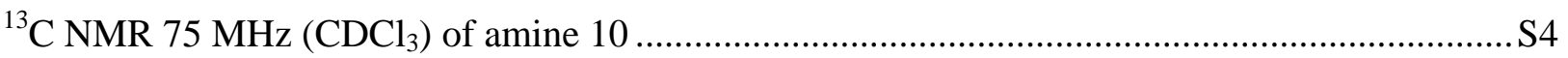

${ }^{1} \mathrm{H}$ NMR $200 \mathrm{MHz}\left(\mathrm{CDCl}_{3}\right)$ of allene 11

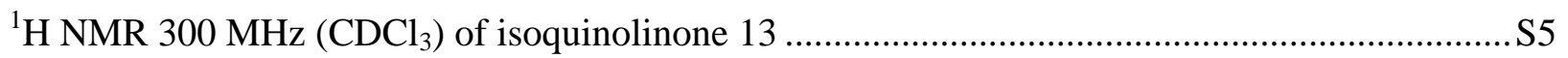

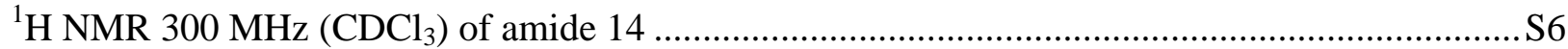

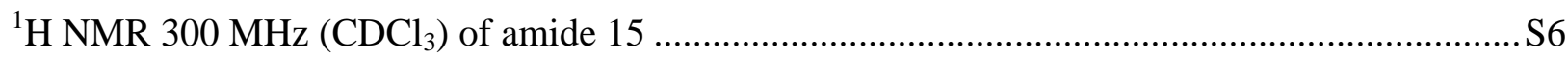

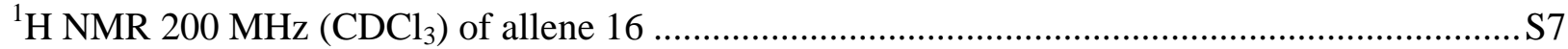

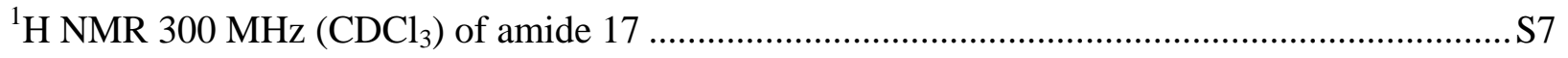

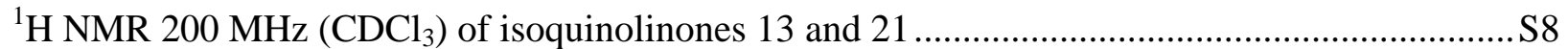

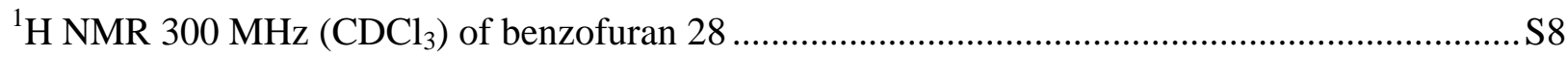

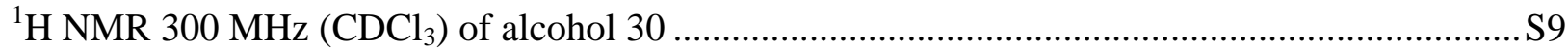

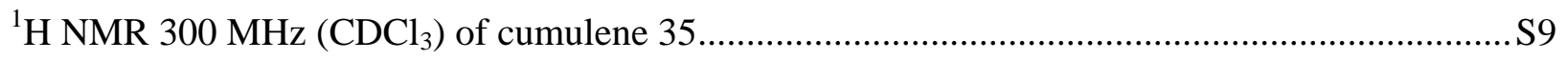

${ }^{1} \mathrm{H}$ NMR $200 \mathrm{MHz}\left(\mathrm{CDCl}_{3}\right)$ chromene 37 and alkyne 36 ....................................................... 10

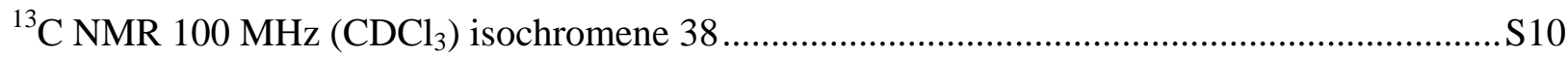

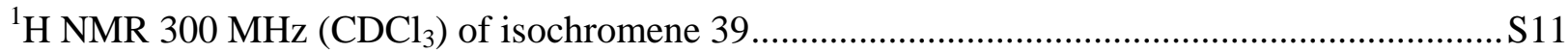

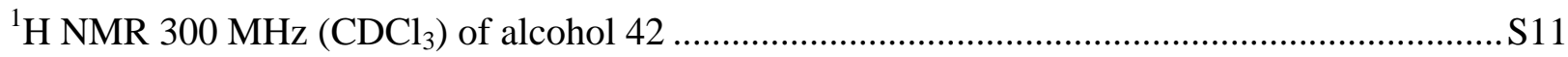

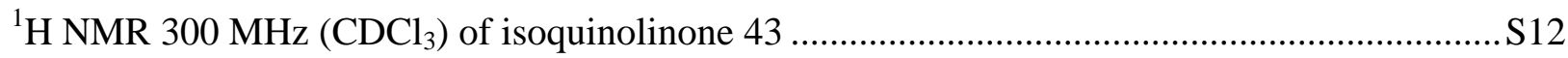

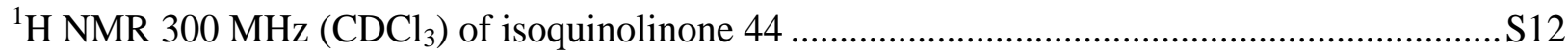

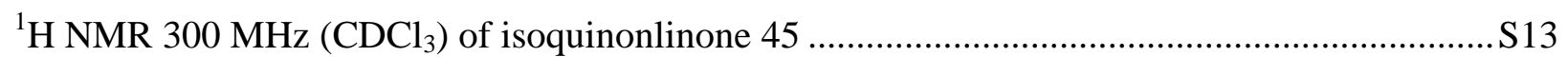

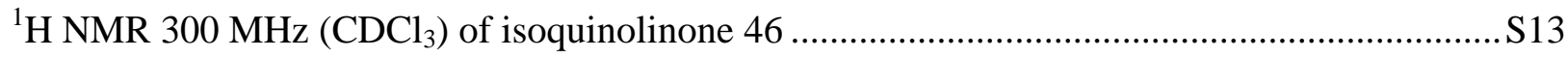

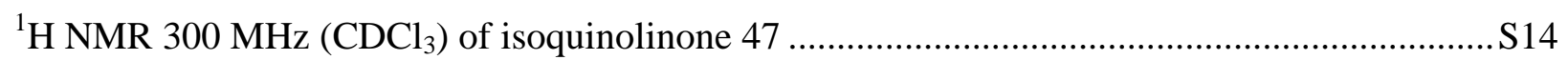

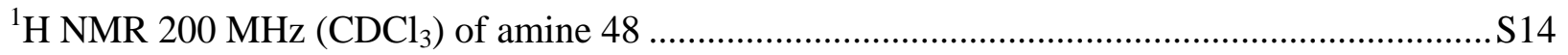


General Aspects. ${ }^{1} \mathrm{H}$ NMR spectra were recorded at 200 or $300 \mathrm{MHz}$ and ${ }^{13} \mathrm{C}$ NMR spectra at $75 \mathrm{MHz}$; chemical shift $(\delta)$ are given in parts per million (ppm) and the coupling constants $(J)$ in hertz. The solvent was deuteriochloroform. IR spectra were recorded by transmission. Gas chromatography analyses were performed on a high-resolution DB-1 type column (30 m $\times 0.25 \mathrm{~mm}$ i.d., $0.25 \mu \mathrm{m}$ coating). GC/MS analyses were performed on an instrument equipped with the same column. The mass spectra under electron impact conditions (EI) were recorded at $70 \mathrm{eV}$ ionizing potential; methane $\left(\mathrm{CH}_{4}\right)$ and ammonia $\left(\mathrm{NH}_{3}\right)$ were used for chemical ionization (CI). The silica gel used for column chromatography was 230-400 mesh. All reagents were reagent grade and were used as such or distilled prior to use. Ethers were distilled from sodium/benzophenone, (chlorinated) hydrocarbons and $\mathrm{Et}_{3} \mathrm{~N}$ from $\mathrm{CaH}_{2}$. All reactions were magnetically stirred. 
${ }^{1} \mathrm{H}$ NMR $300 \mathrm{MHz}\left(\mathrm{CDCl}_{3}\right)$ of alkyne 3

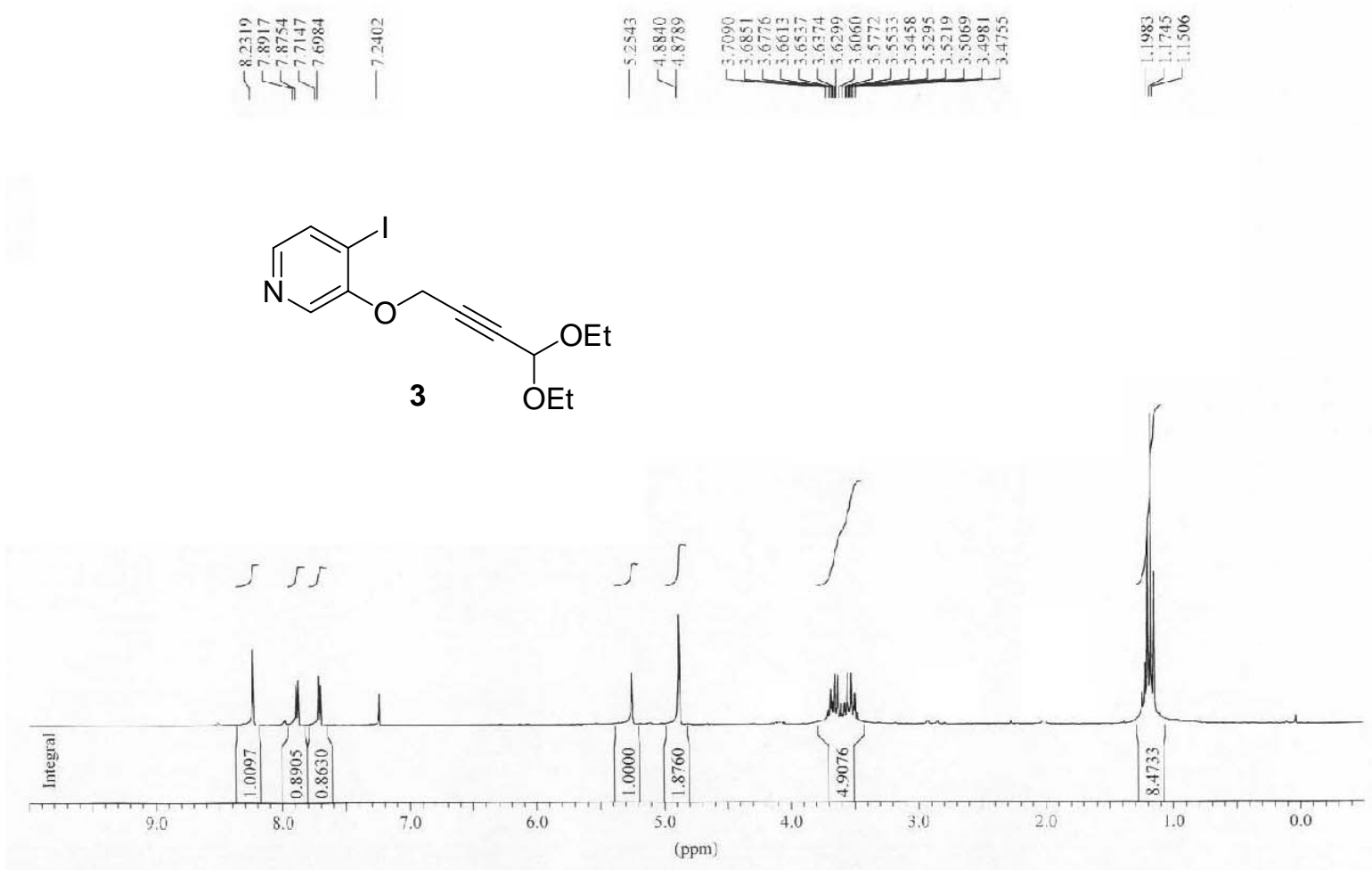

${ }^{1} \mathrm{H}$ NMR $200 \mathrm{MHz}\left(\mathrm{CDCl}_{3}\right)$ of allene 4
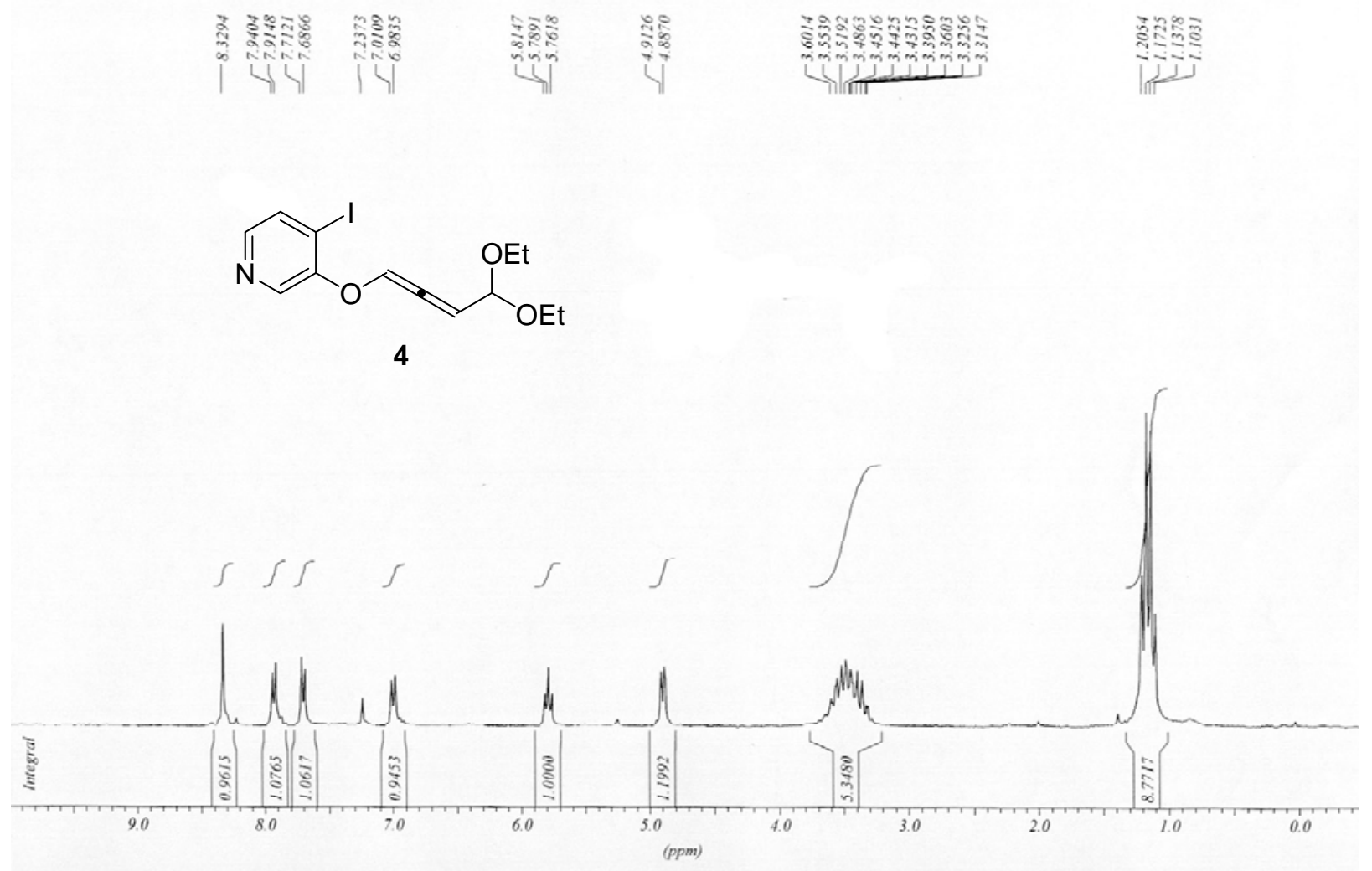
${ }^{1} \mathrm{H}$ NMR $200 \mathrm{MHz}\left(\mathrm{CDCl}_{3}\right)$ of furopyridine 6
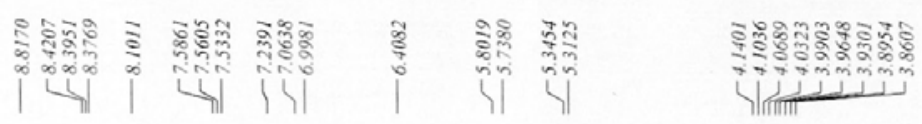<smiles>CCOC=Cc1coc2cnccc12</smiles>
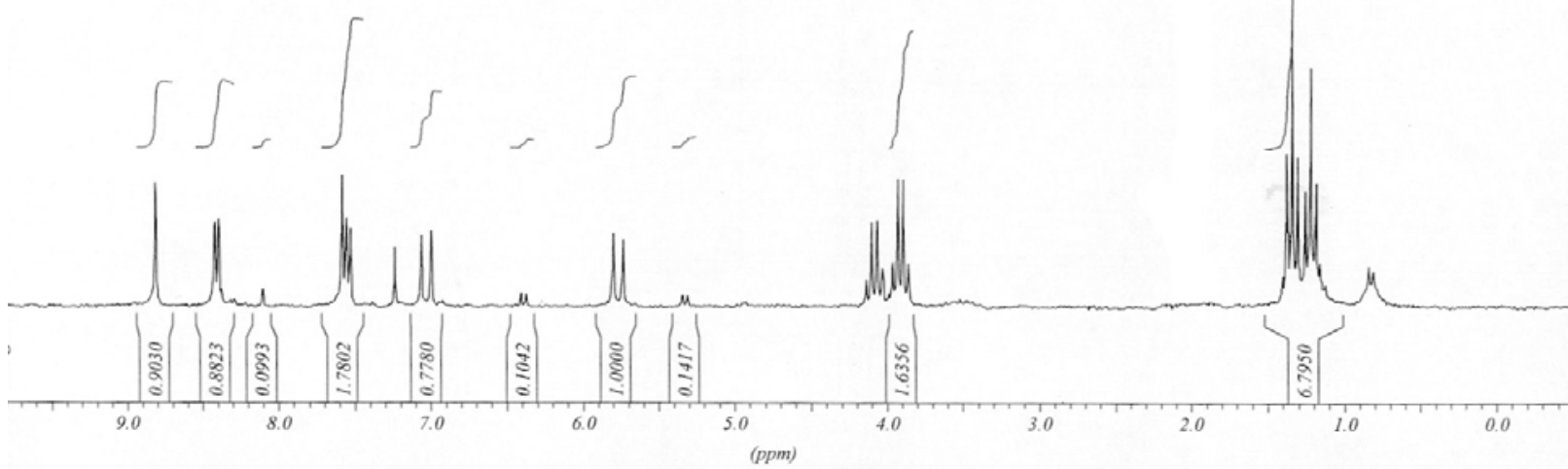

${ }^{13} \mathrm{C}$ NMR $75 \mathrm{MHz}\left(\mathrm{CDCl}_{3}\right)$ of amine 10

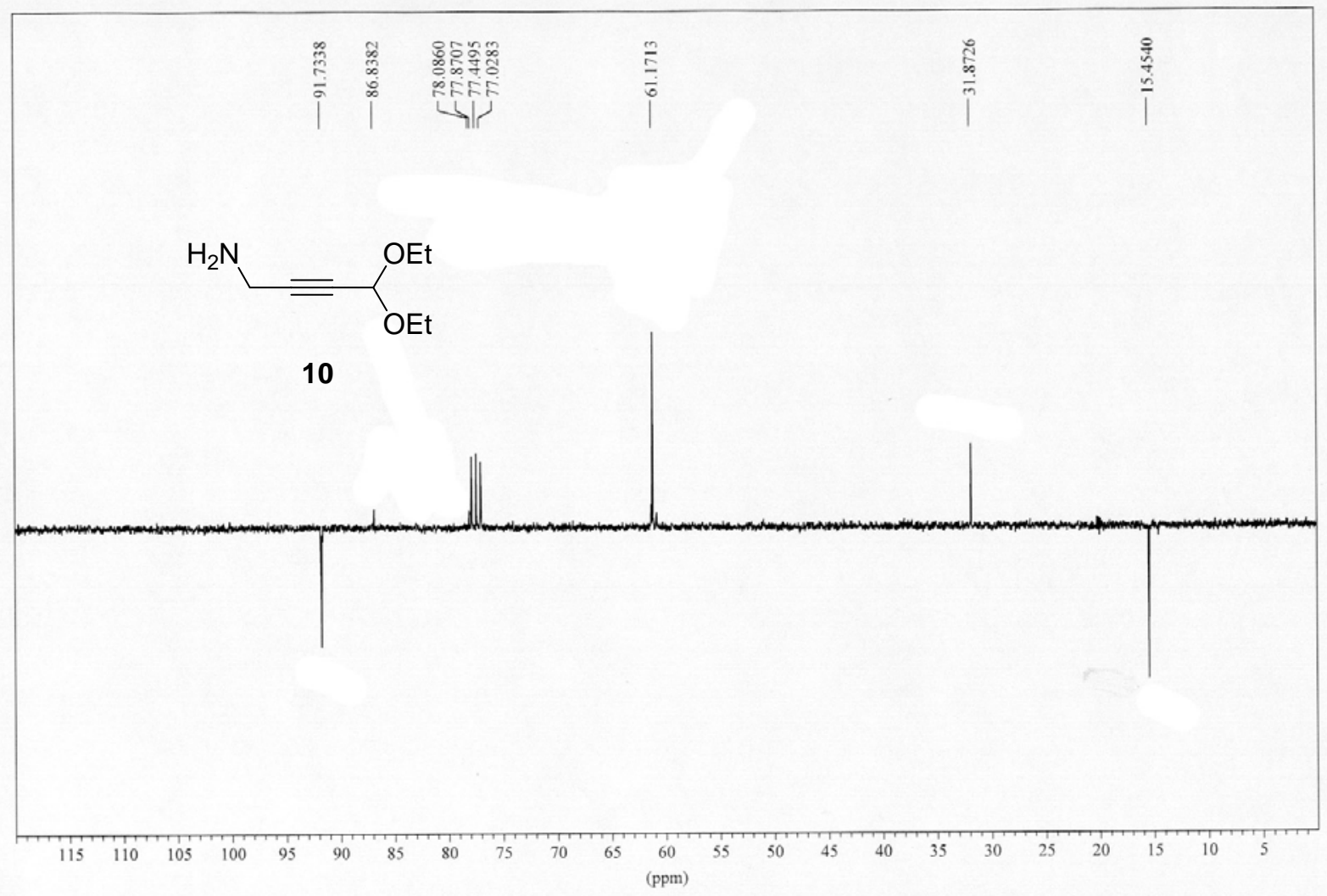


${ }^{1} \mathrm{H}$ NMR $200 \mathrm{MHz}\left(\mathrm{CDCl}_{3}\right)$ of allene 11

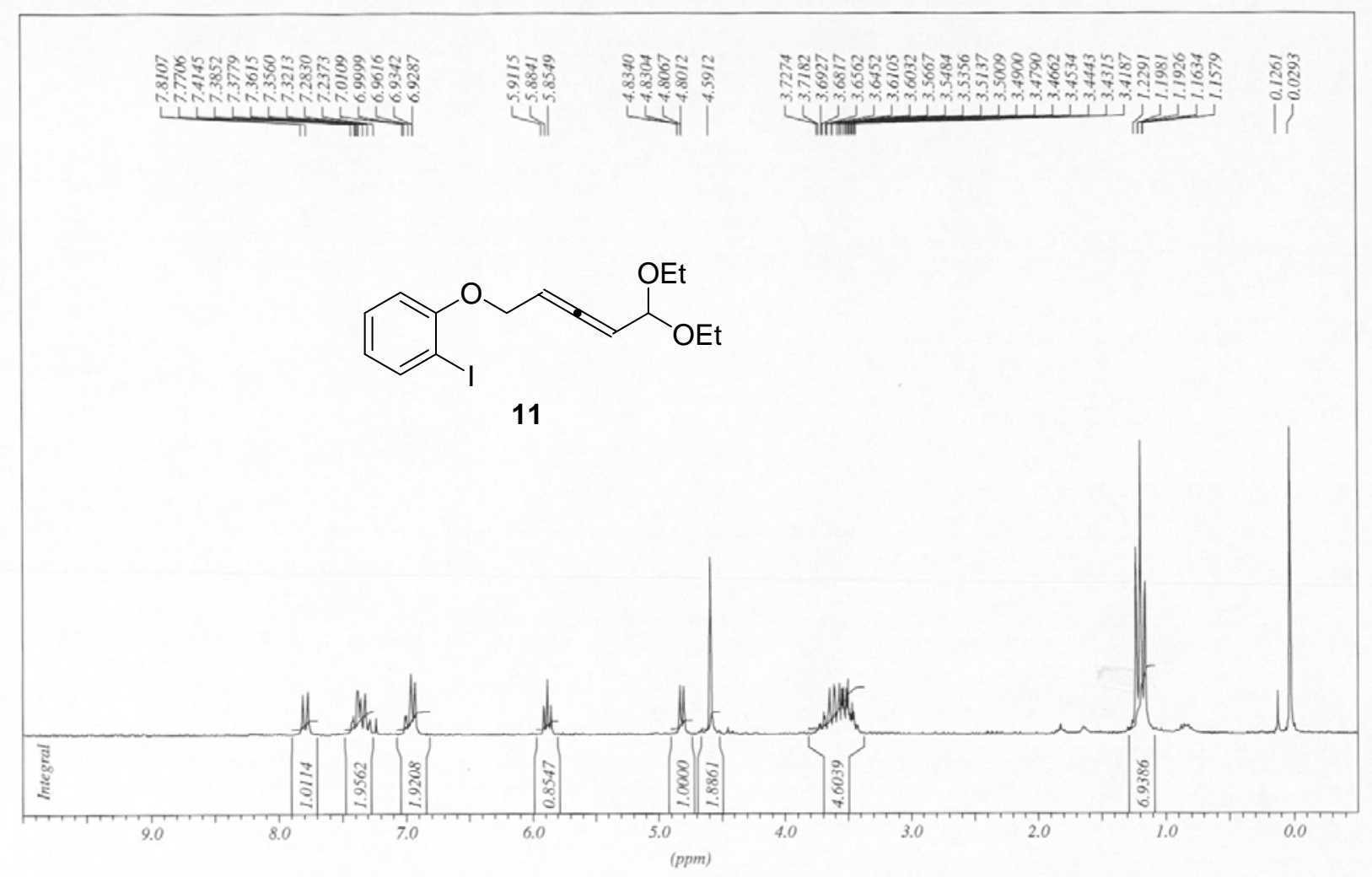

${ }^{1} \mathrm{H}$ NMR $300 \mathrm{MHz}\left(\mathrm{CDCl}_{3}\right)$ of isoquinolinone 13

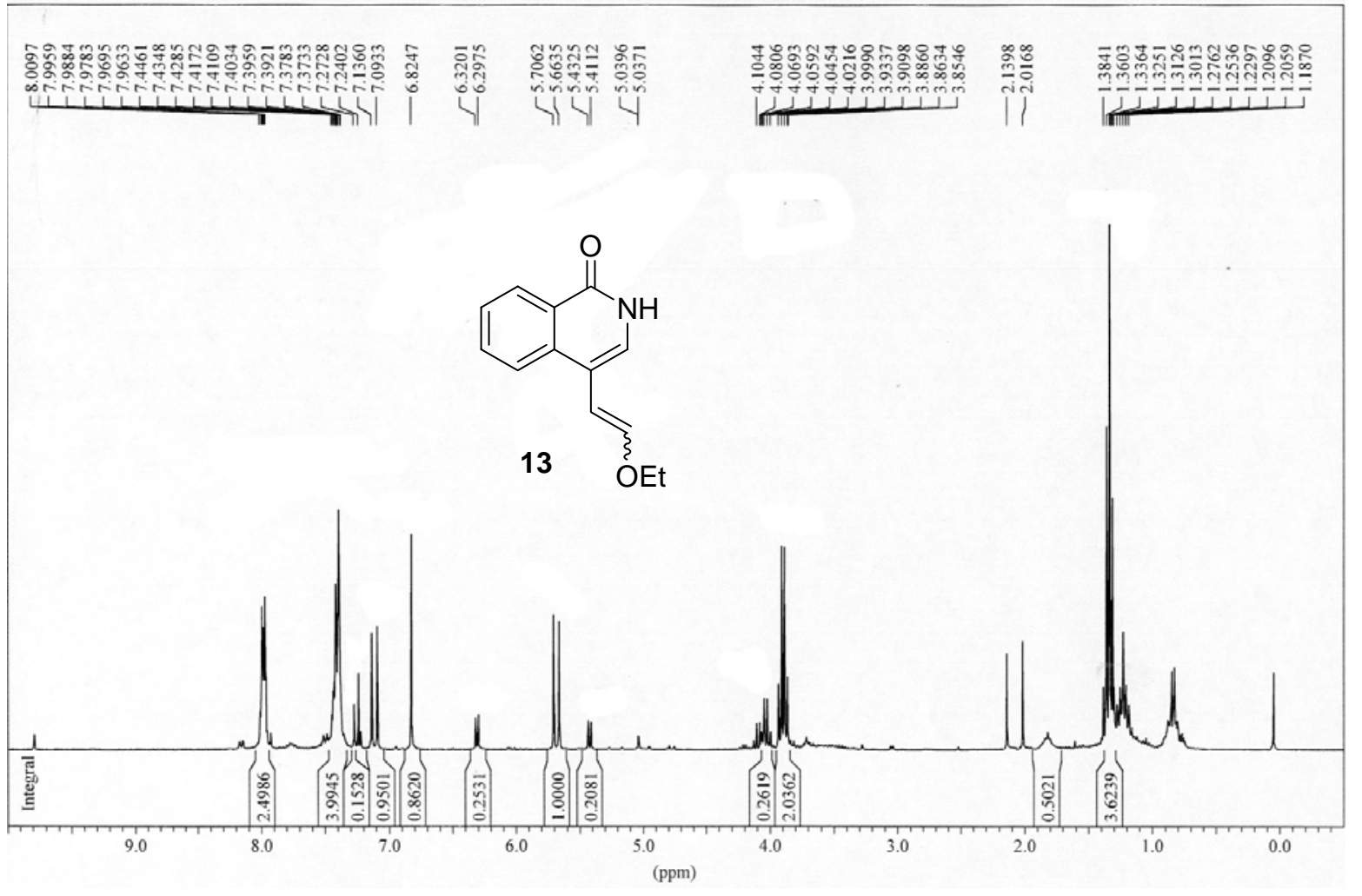


${ }^{1} \mathrm{H}$ NMR $300 \mathrm{MHz}\left(\mathrm{CDCl}_{3}\right)$ of amide $\mathbf{1 4}$

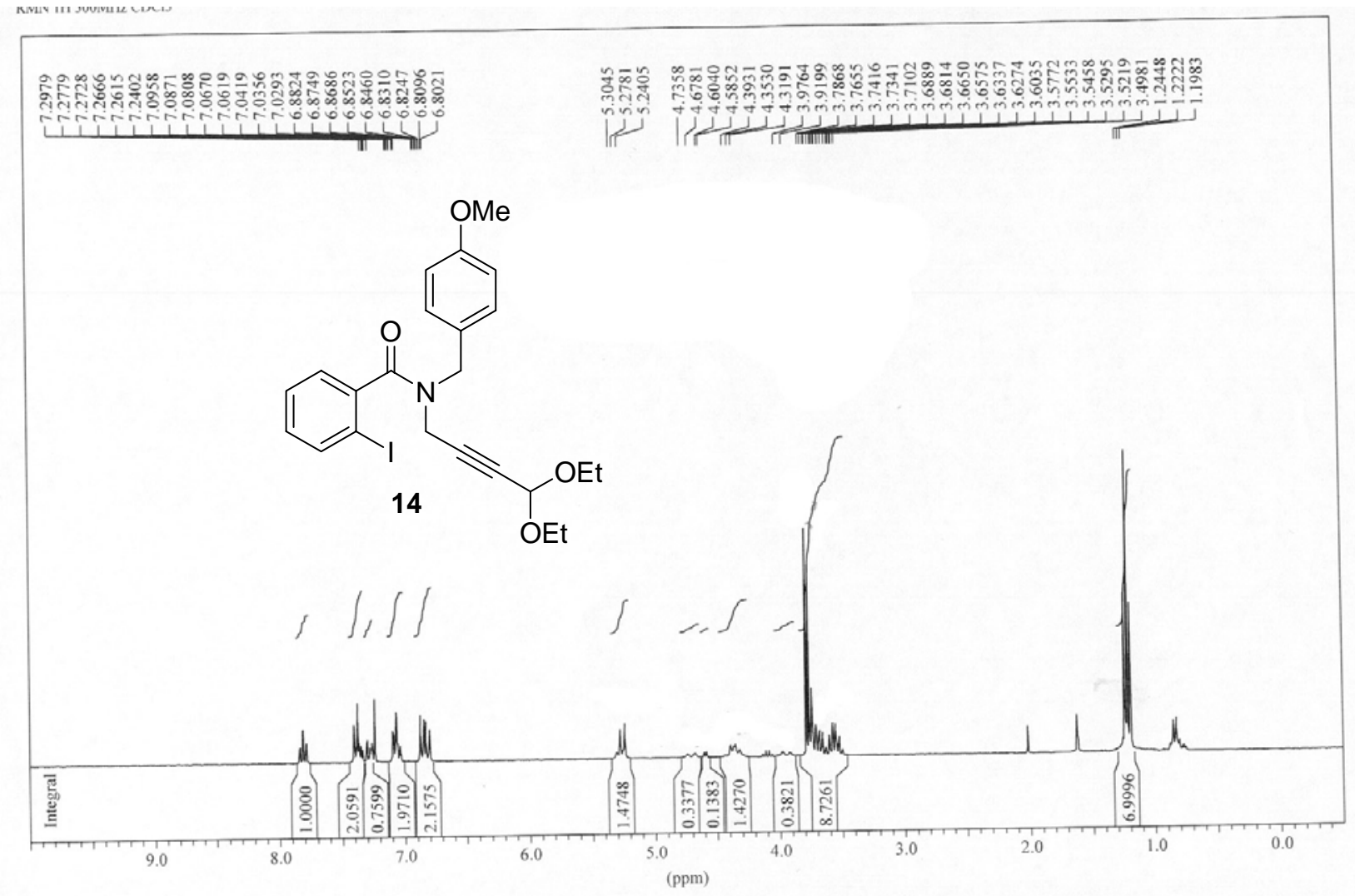

${ }^{1} \mathrm{H}$ NMR $300 \mathrm{MHz}\left(\mathrm{CDCl}_{2}\right)$ of amide 15

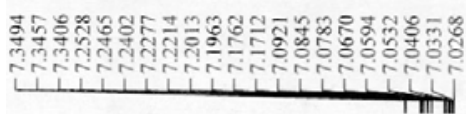

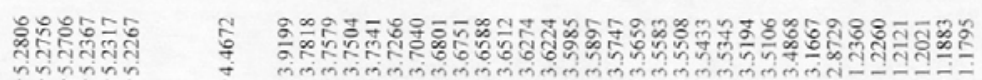

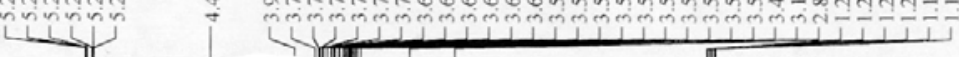

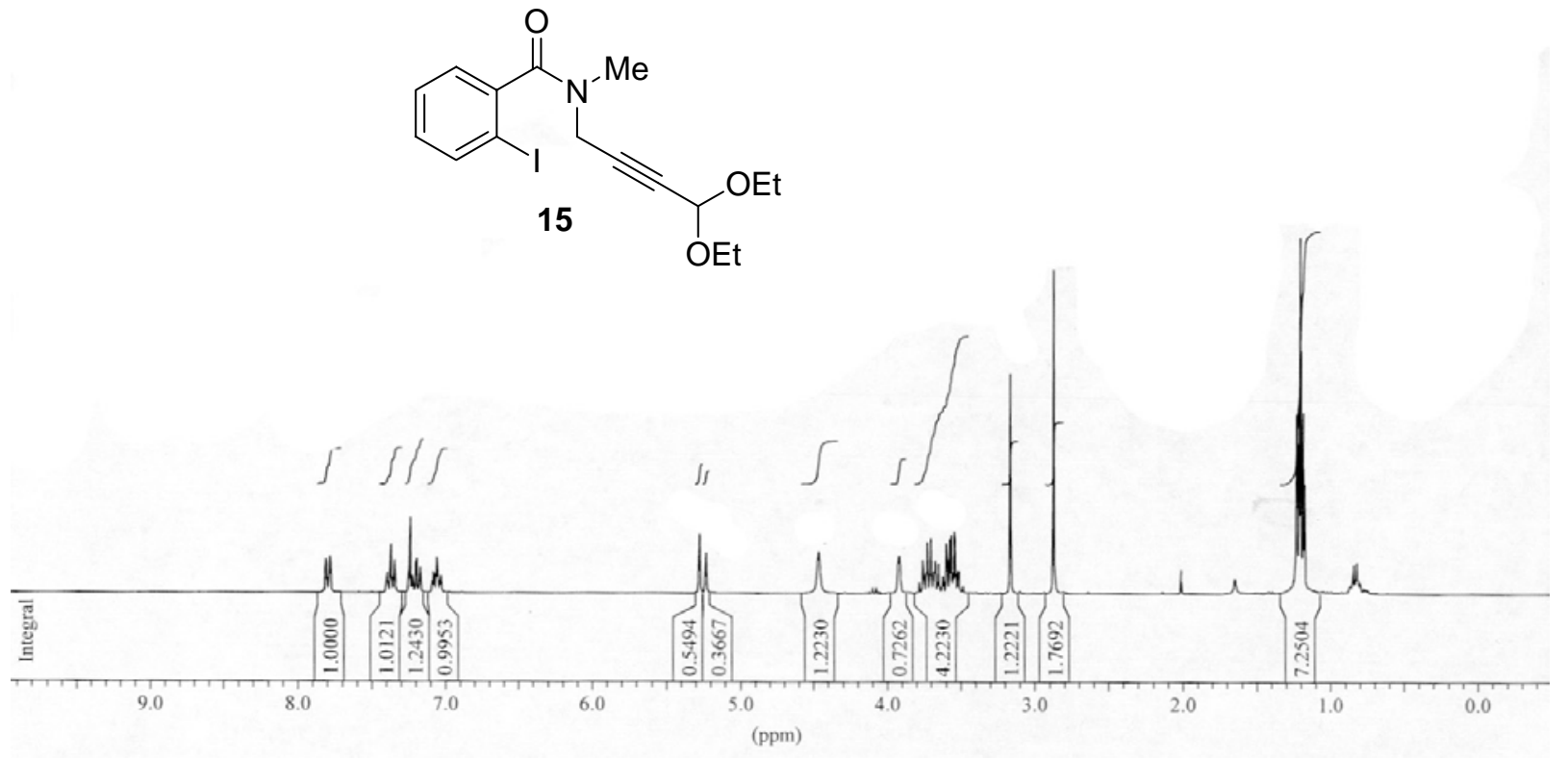


${ }^{1} \mathrm{H}$ NMR $200 \mathrm{MHz}\left(\mathrm{CDCl}_{3}\right)$ of allene 16

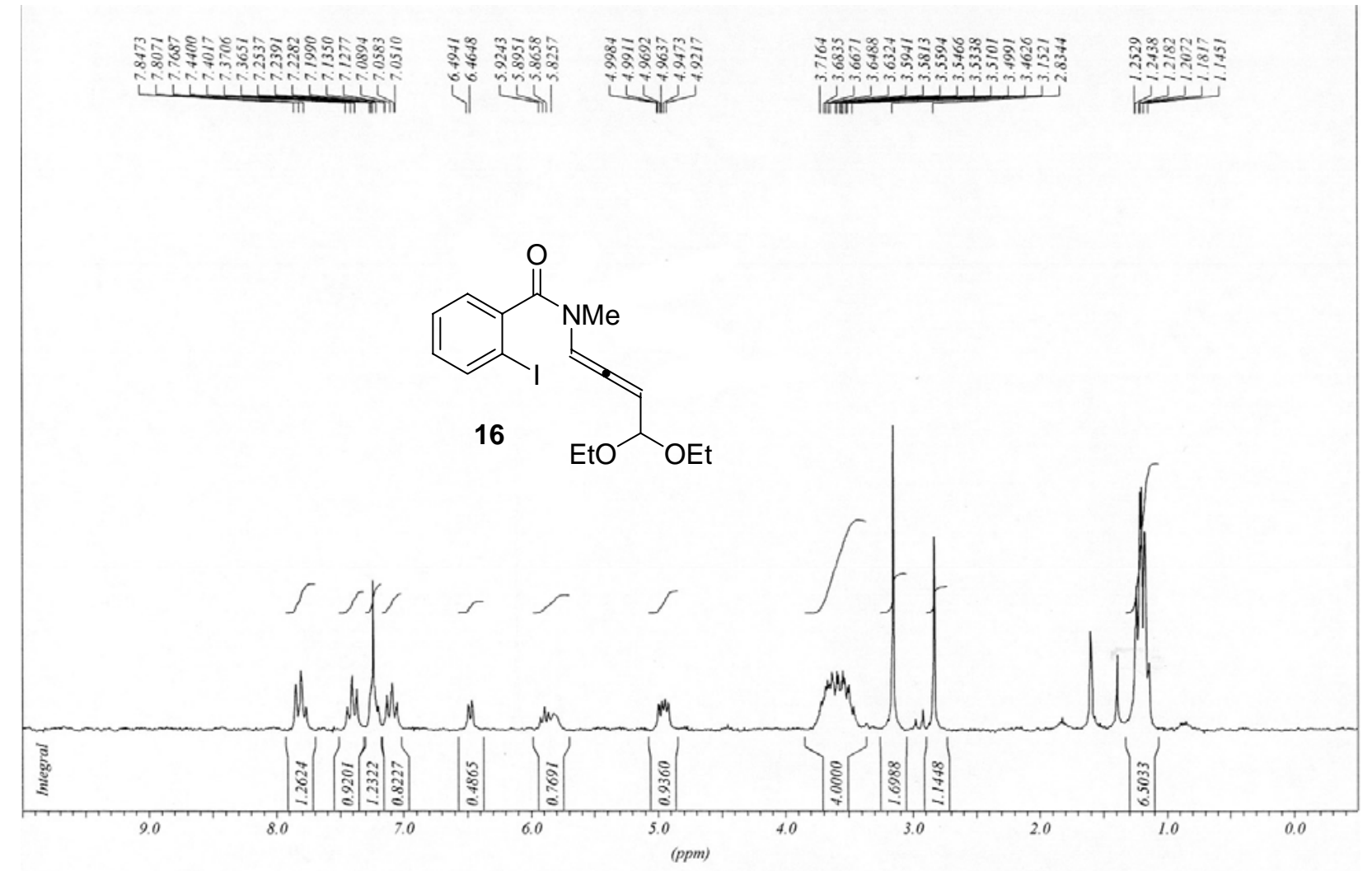

${ }^{1} \mathrm{H}$ NMR $300 \mathrm{MHz}\left(\mathrm{CDCl}_{3}\right)$ of amide $\mathbf{1 7}$

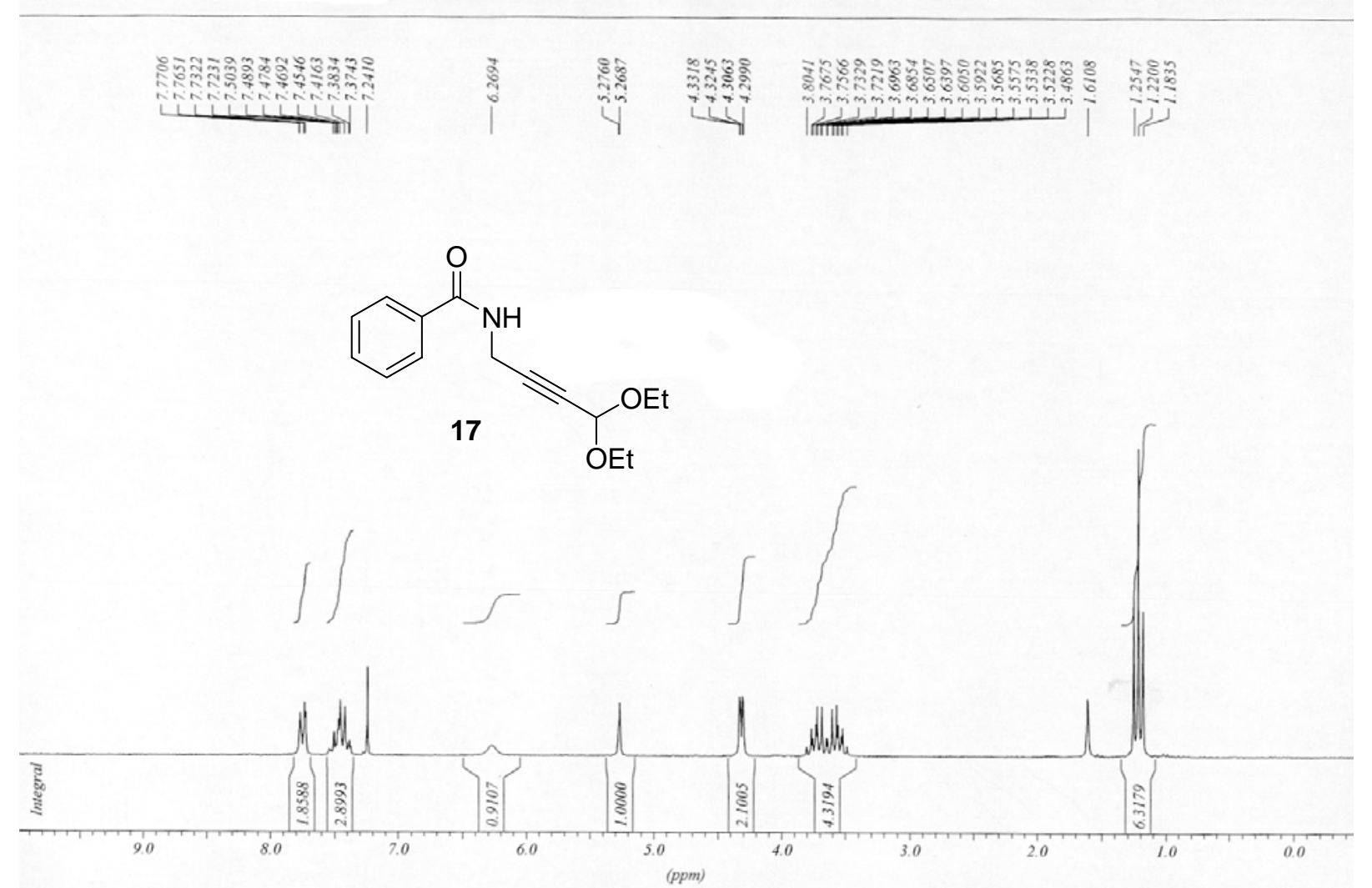


${ }^{1} \mathrm{H}$ NMR $200 \mathrm{MHz}\left(\mathrm{CDCl}_{3}\right)$ of isoquinolinones 13 and 21
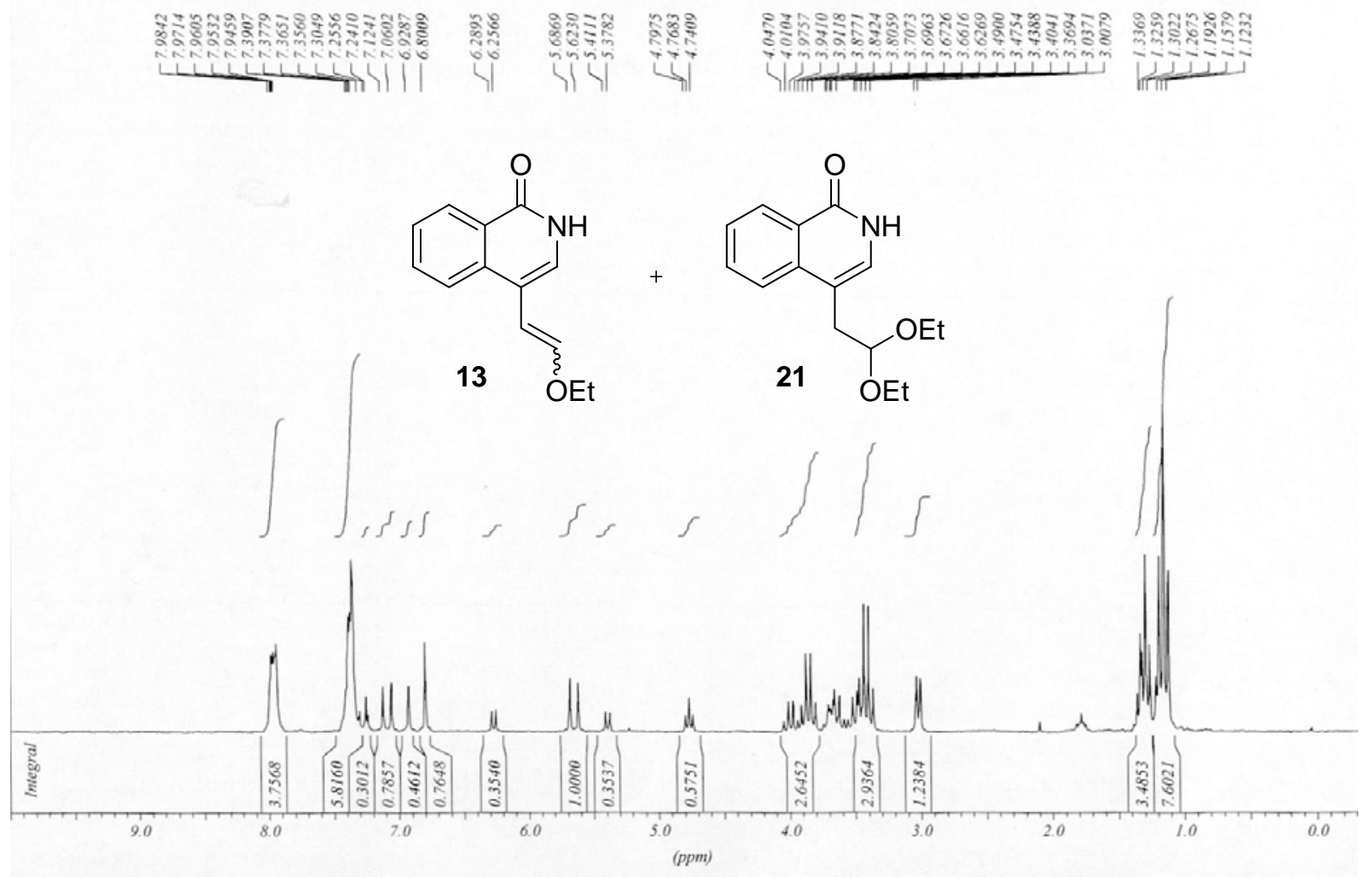

${ }^{1} \mathrm{H}$ NMR $300 \mathrm{MHz}\left(\mathrm{CDCl}_{3}\right)$ of benzofuran 28

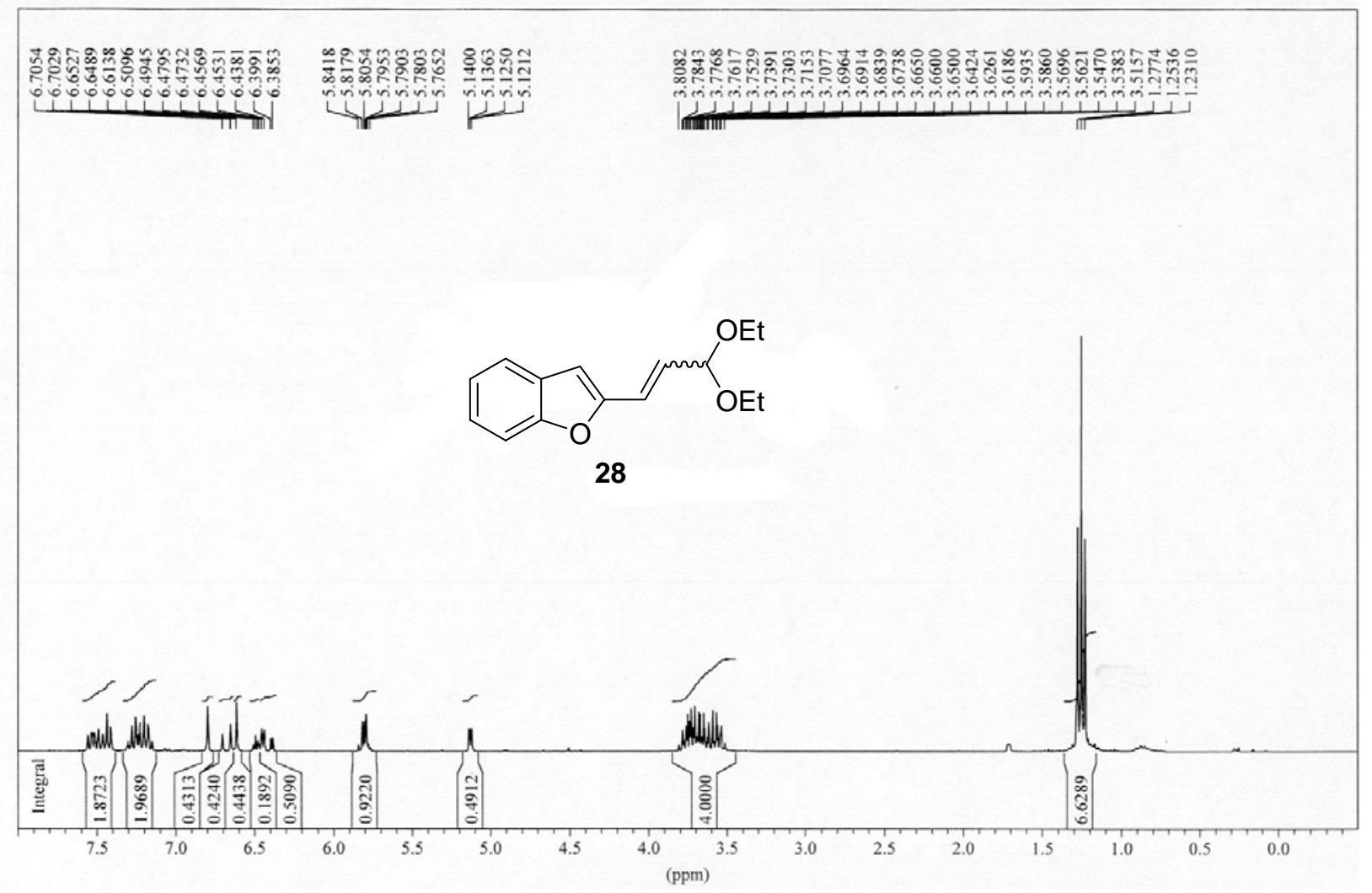


${ }^{1} \mathrm{H}$ NMR $300 \mathrm{MHz}\left(\mathrm{CDCl}_{3}\right)$ of alcohol 30

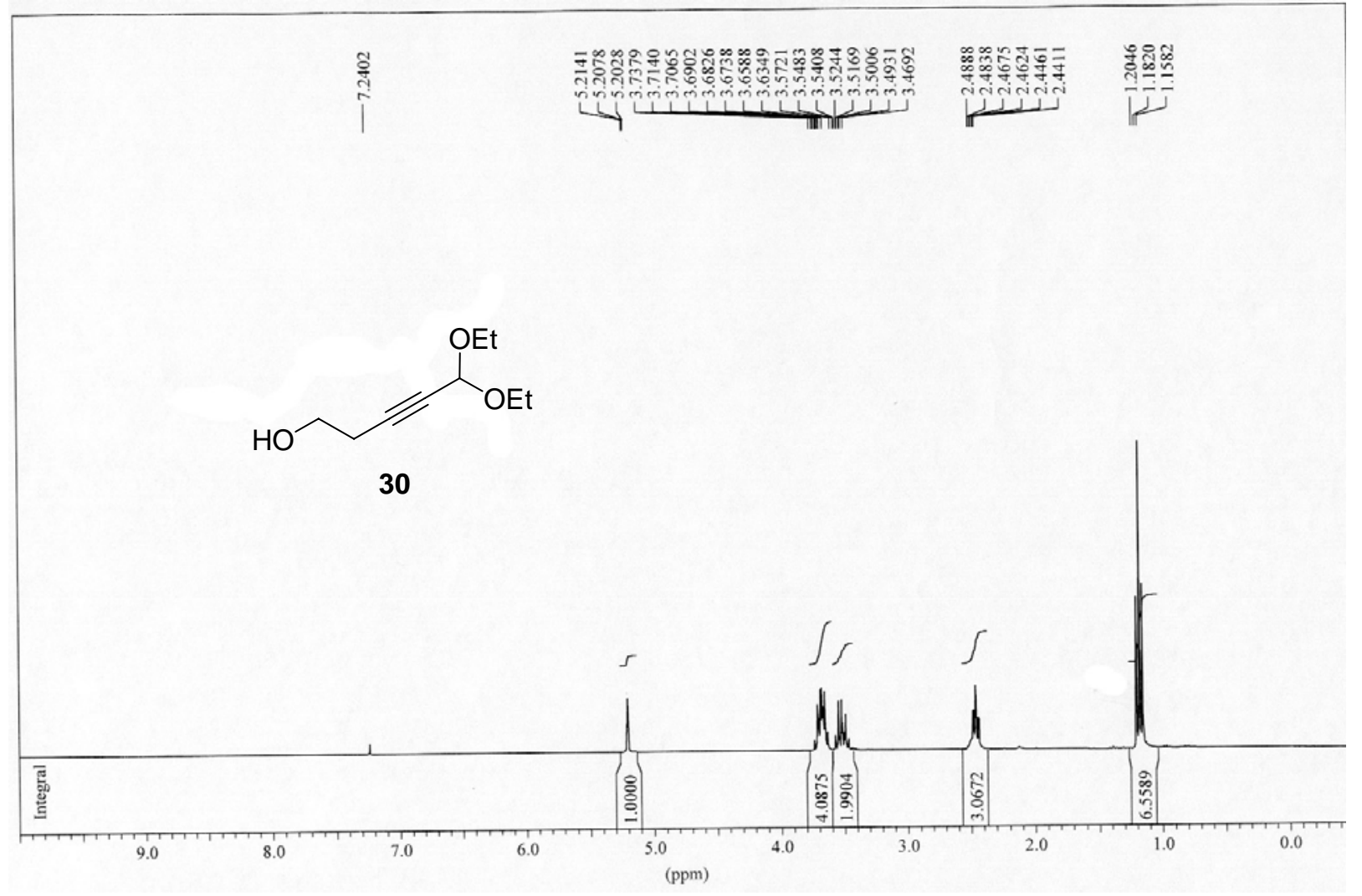

${ }^{1} \mathrm{H}$ NMR $300 \mathrm{MHz}\left(\mathrm{CDCl}_{3}\right)$ of cumulene 35

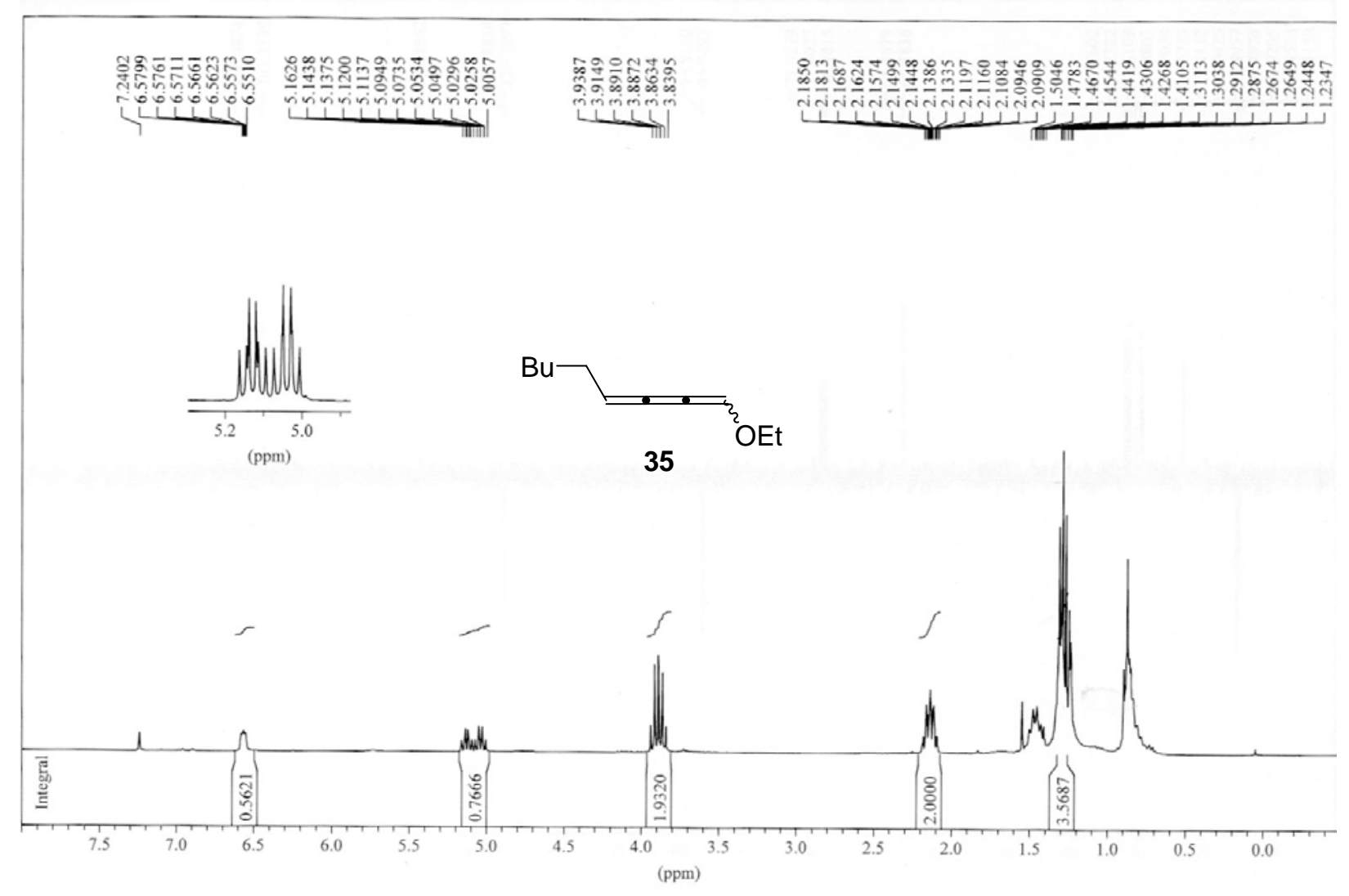


${ }^{1} \mathrm{H}$ NMR $200 \mathrm{MHz}\left(\mathrm{CDCl}_{3}\right)$ chromene 37 and alkyne 36

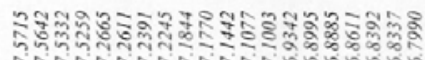

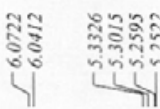

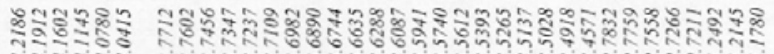

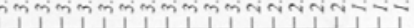<smiles>CCOC(C=C1CCOc2ccccc21)OCC</smiles><smiles>CCOC(C#CCCOc1ccccc1)OCC</smiles>

36

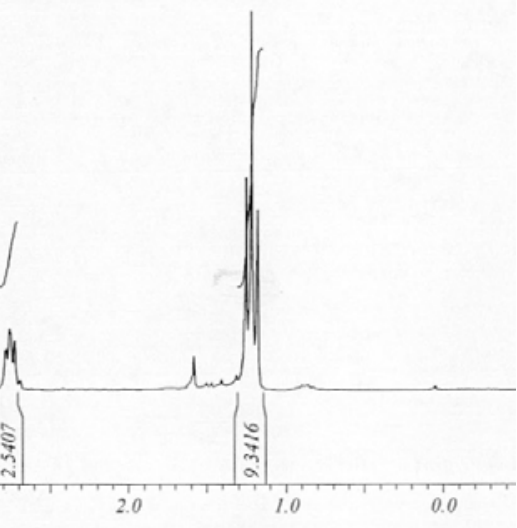

${ }^{13} \mathrm{C}$ NMR $100 \mathrm{MHz}\left(\mathrm{CDCl}_{3}\right)$ isochromene 38

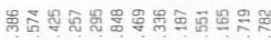

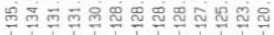

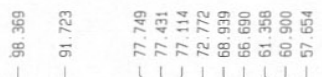

(n)
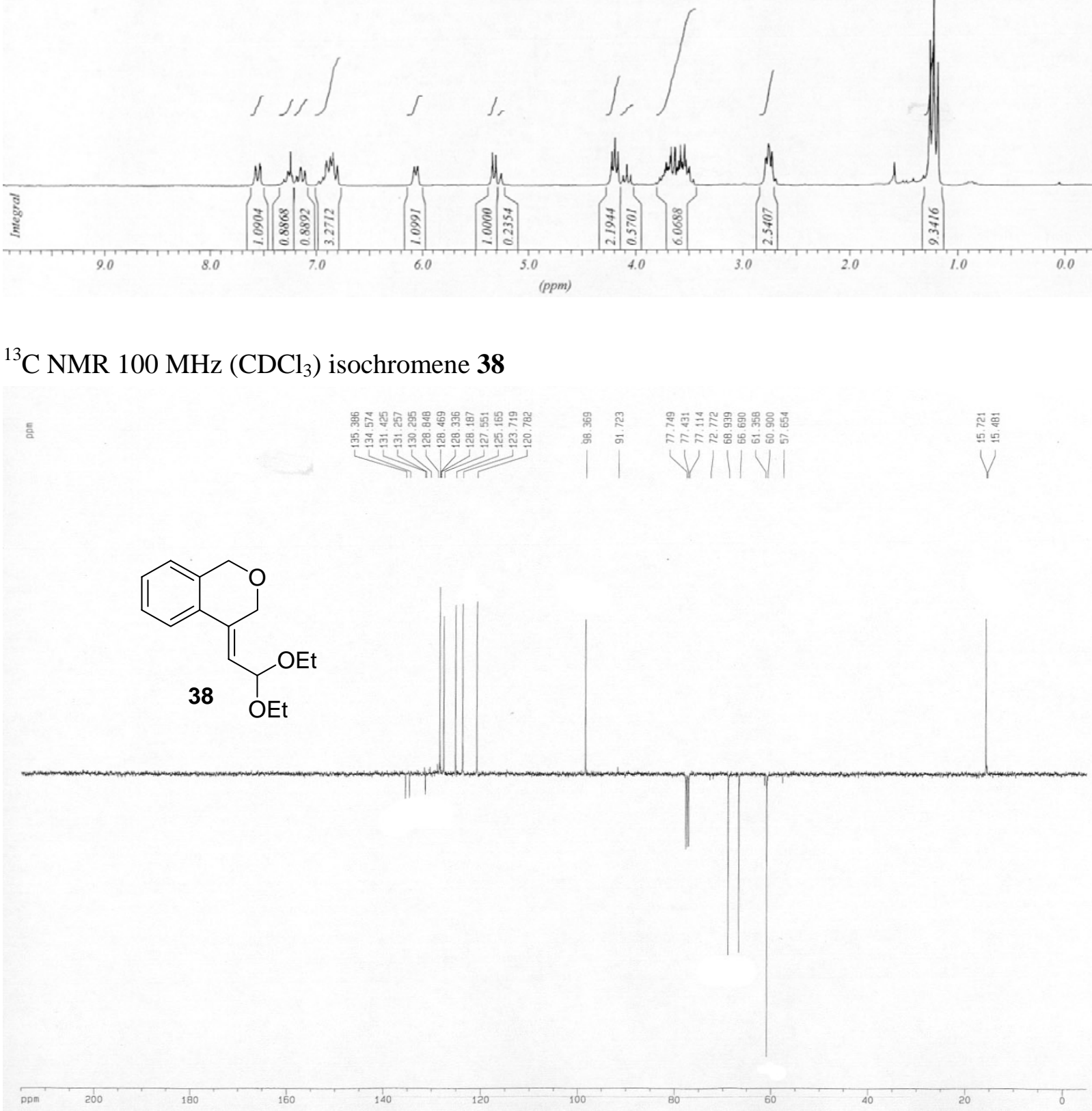
${ }^{1} \mathrm{H}$ NMR $300 \mathrm{MHz}\left(\mathrm{CDCl}_{3}\right)$ of isochromene 39
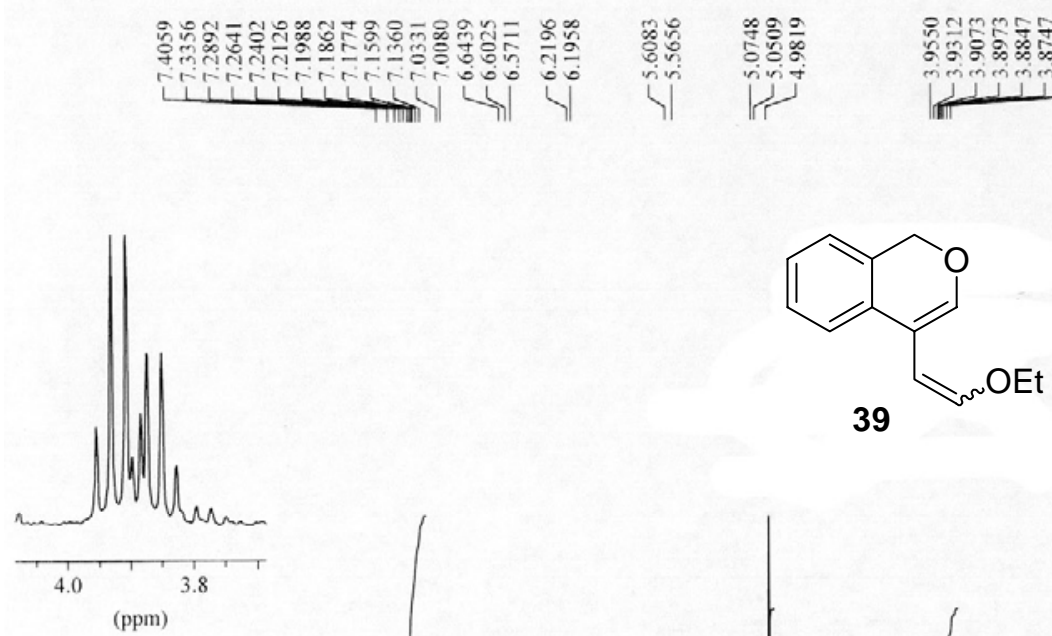

39

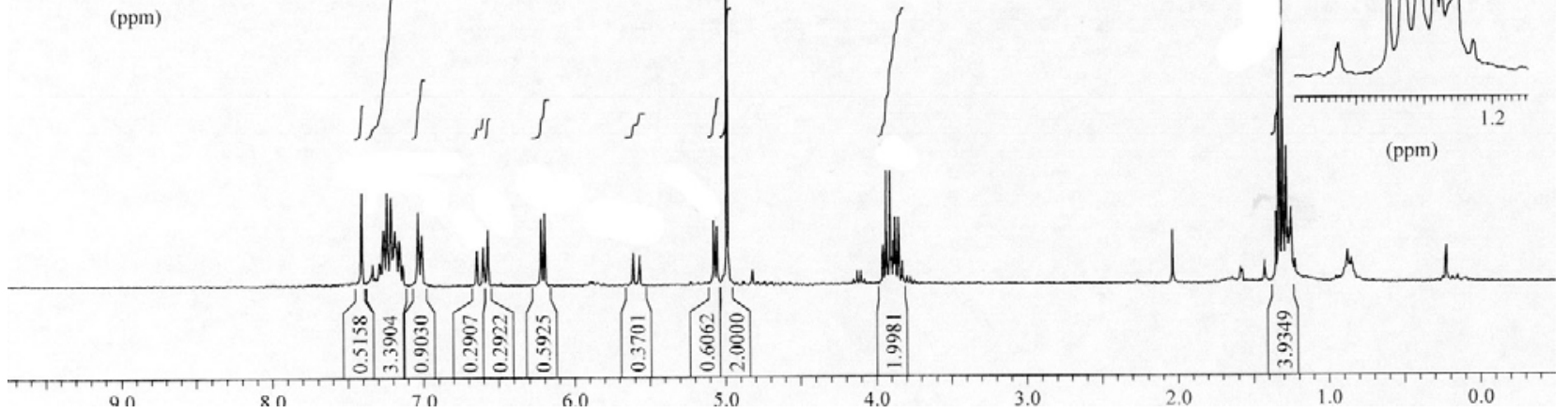

${ }^{1} \mathrm{H}$ NMR $300 \mathrm{MHz}\left(\mathrm{CDCl}_{3}\right)$ of alcohol 42

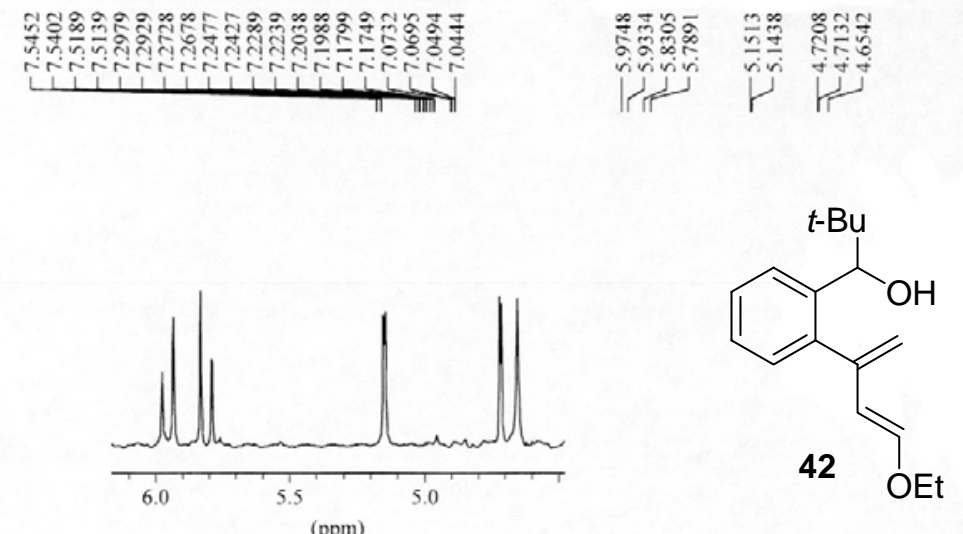

$1 \int 1$

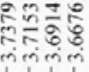

|ं

$\int$
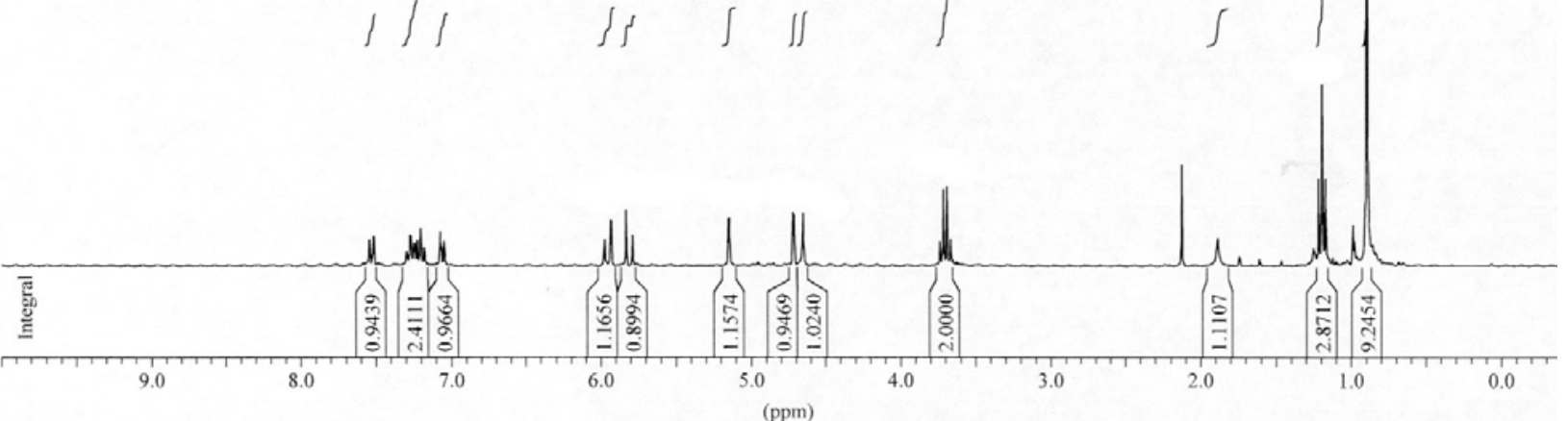
${ }^{1} \mathrm{H}$ NMR $300 \mathrm{MHz}\left(\mathrm{CDCl}_{3}\right)$ of isoquinolinone 43

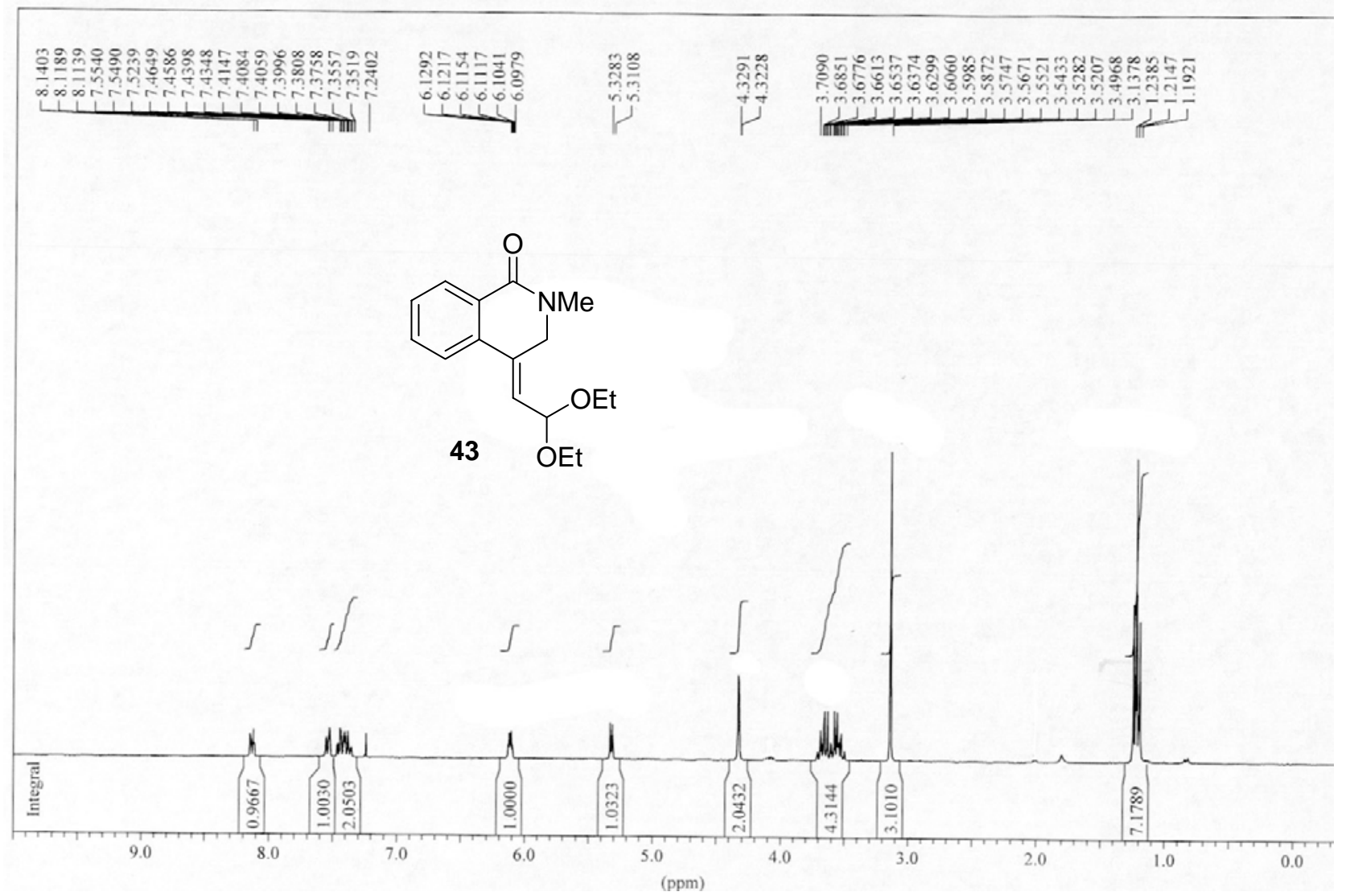

${ }^{1} \mathrm{H}$ NMR $300 \mathrm{MHz}\left(\mathrm{CDCl}_{3}\right)$ of isoquinolinone 44

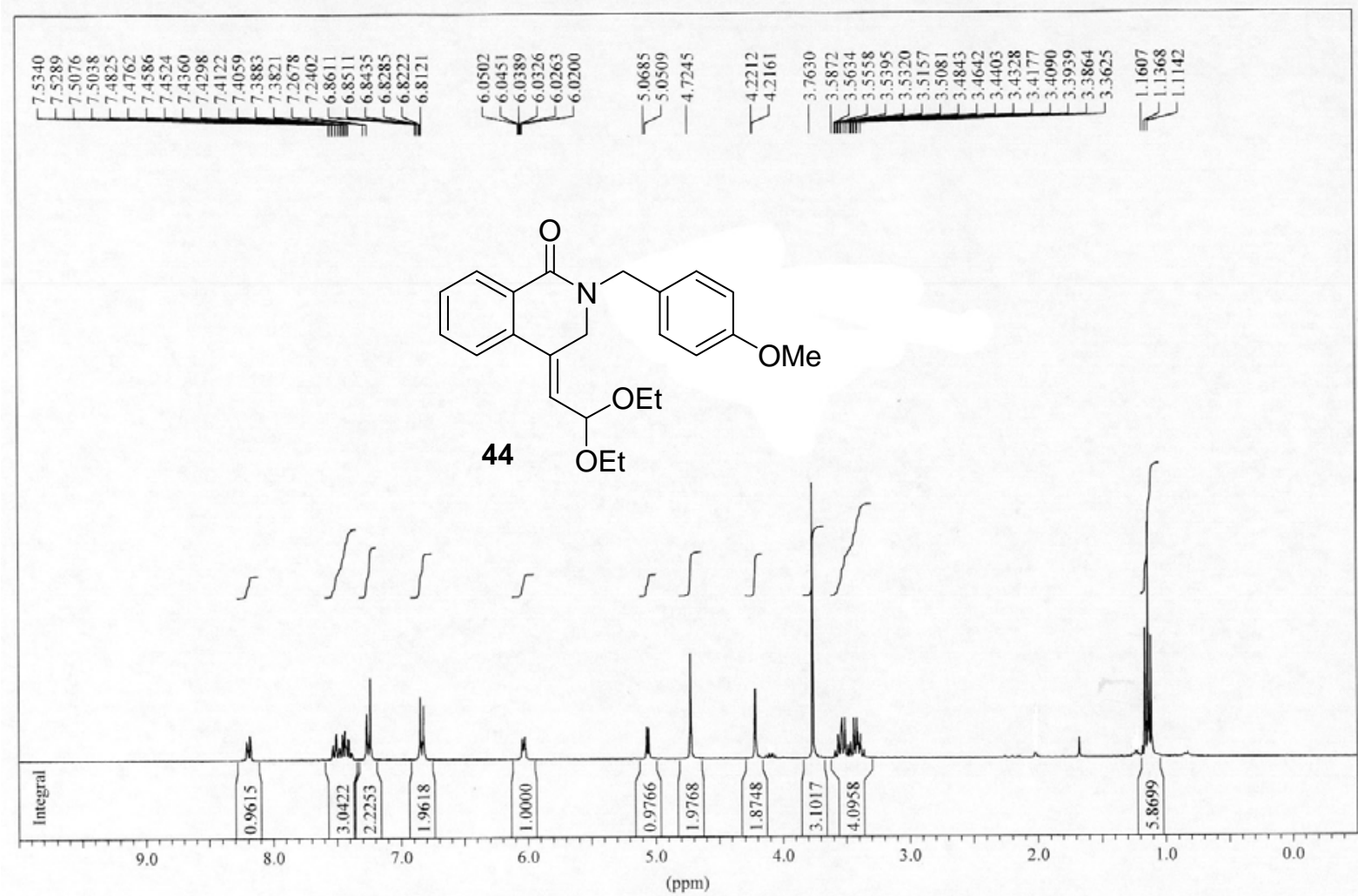


${ }^{1} \mathrm{H}$ NMR $300 \mathrm{MHz}\left(\mathrm{CDCl}_{3}\right)$ of isoquinonlinone 45

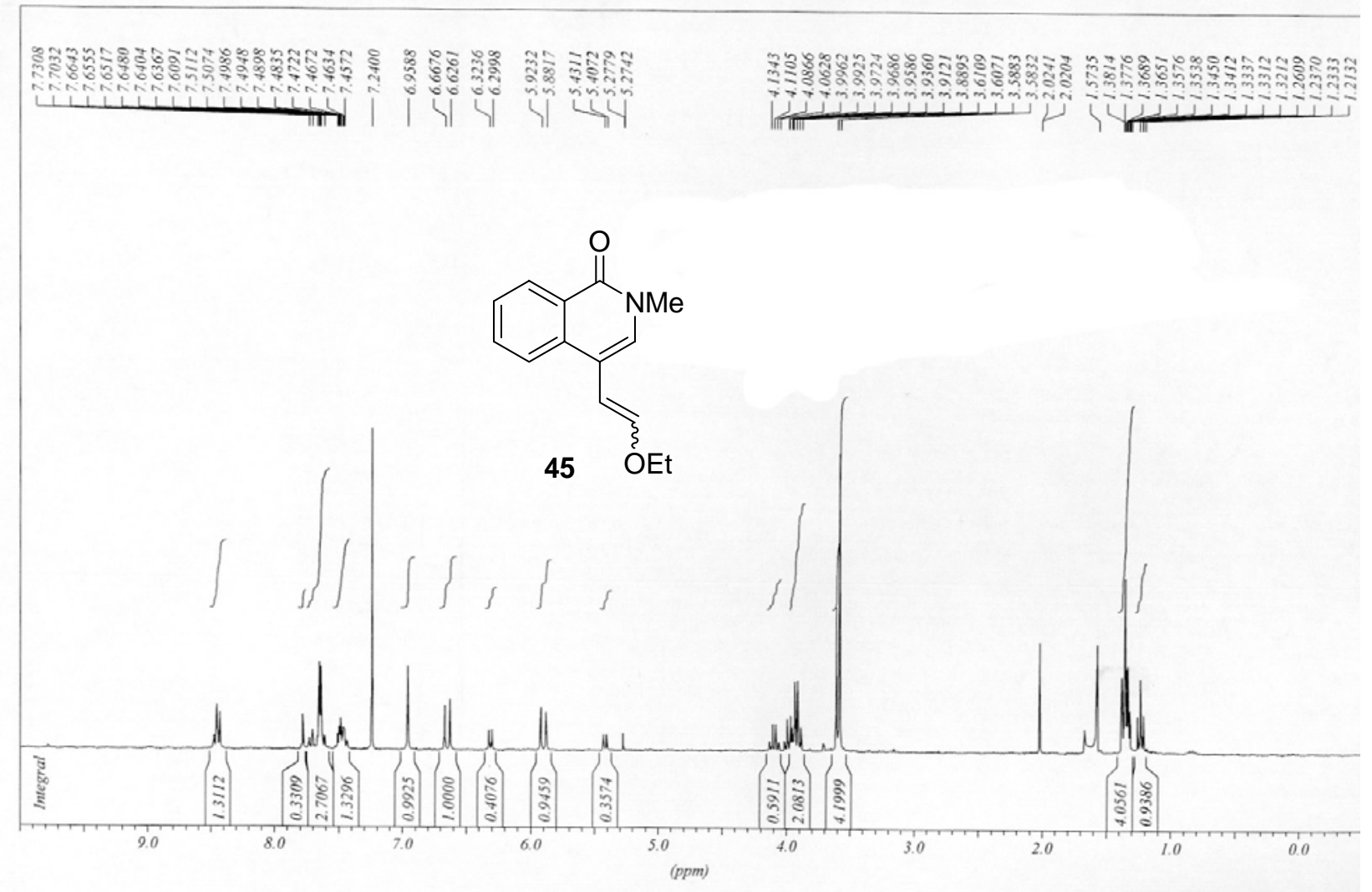

${ }^{1} \mathrm{H}$ NMR $300 \mathrm{MHz}\left(\mathrm{CDCl}_{3}\right)$ of isoquinolinone 46

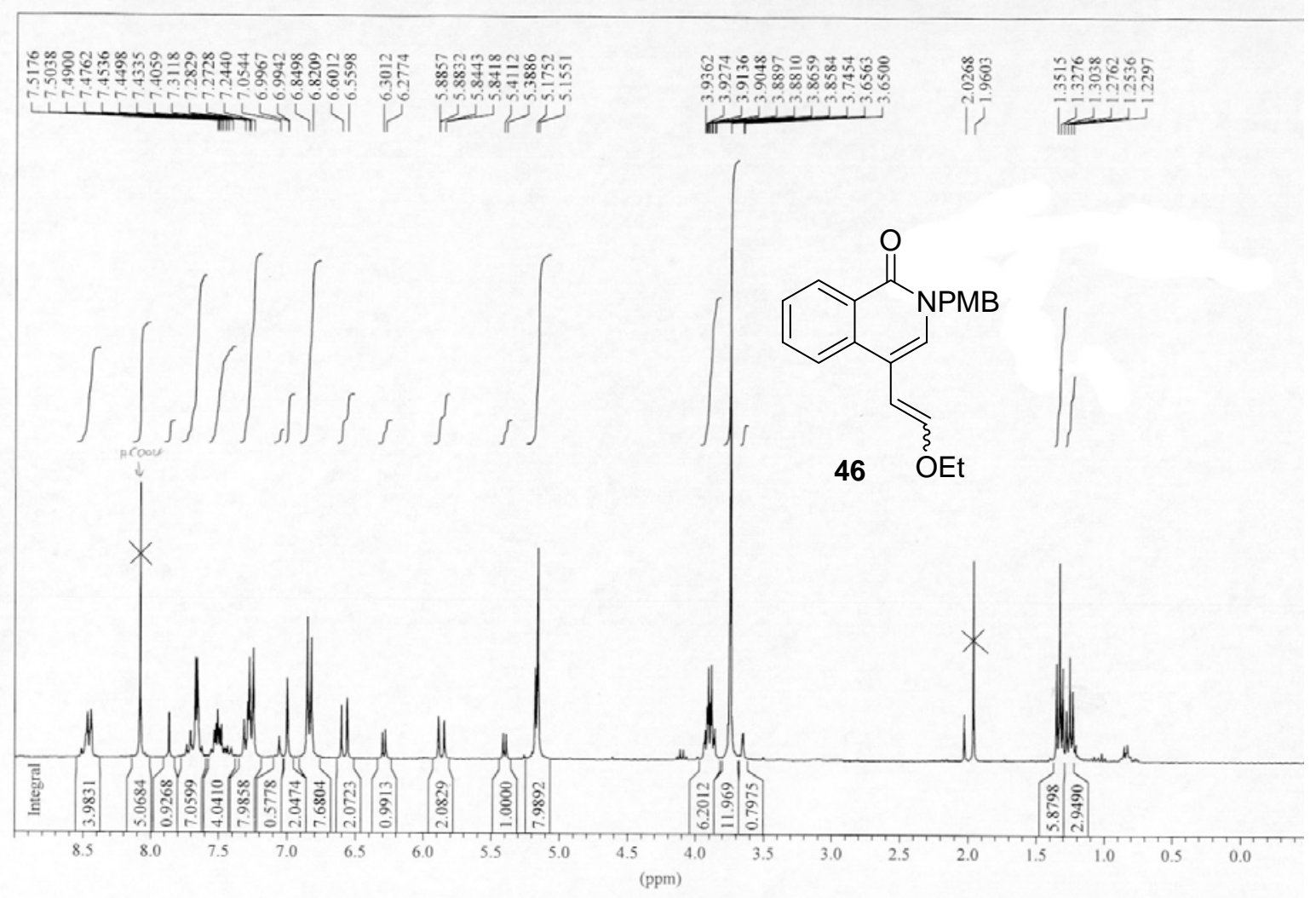


${ }^{1} \mathrm{H}$ NMR $300 \mathrm{MHz}\left(\mathrm{CDCl}_{3}\right)$ of isoquinolinone 47

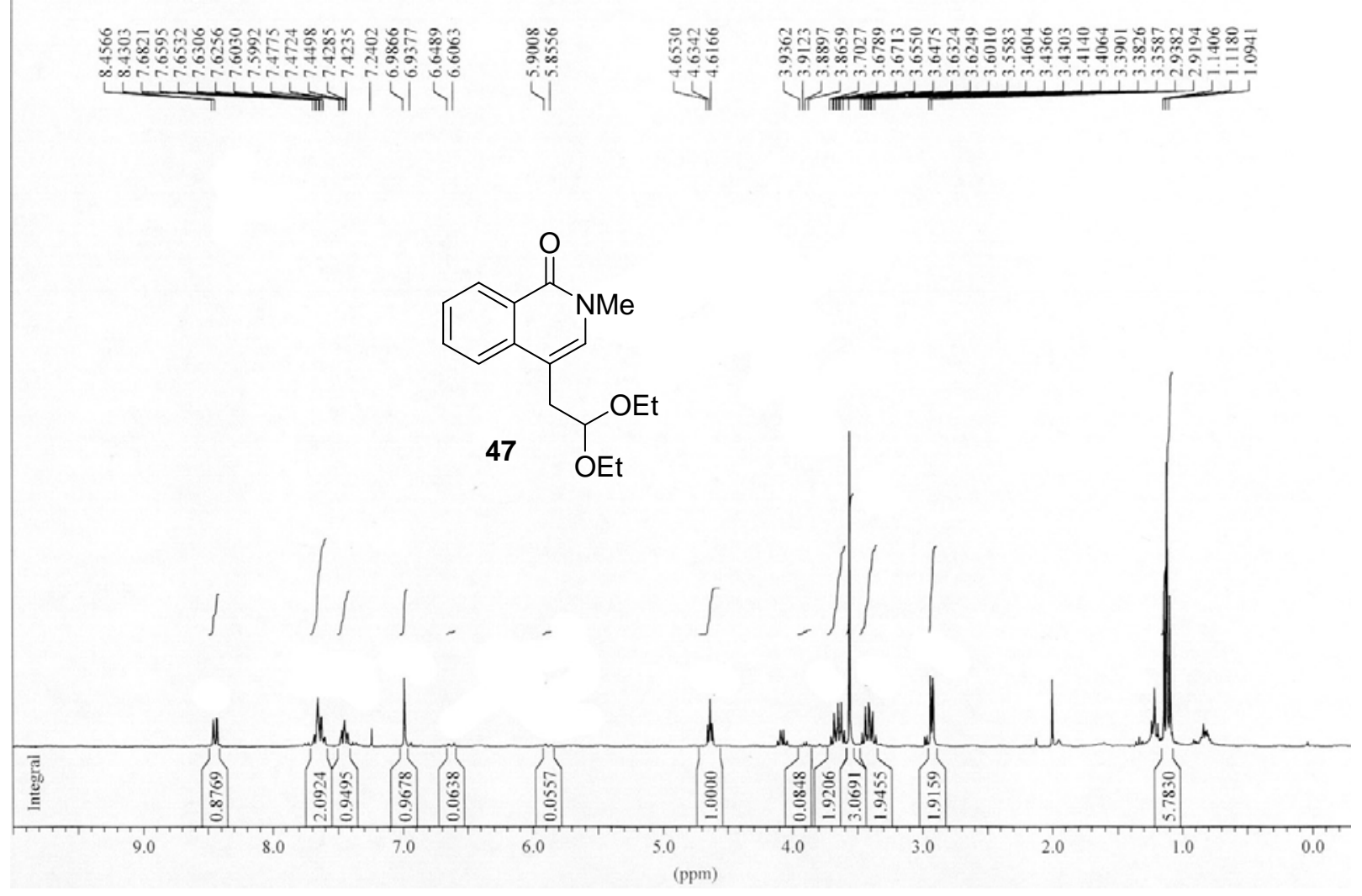

${ }^{1} \mathrm{H}$ NMR $200 \mathrm{MHz}\left(\mathrm{CDCl}_{3}\right)$ of amine 48

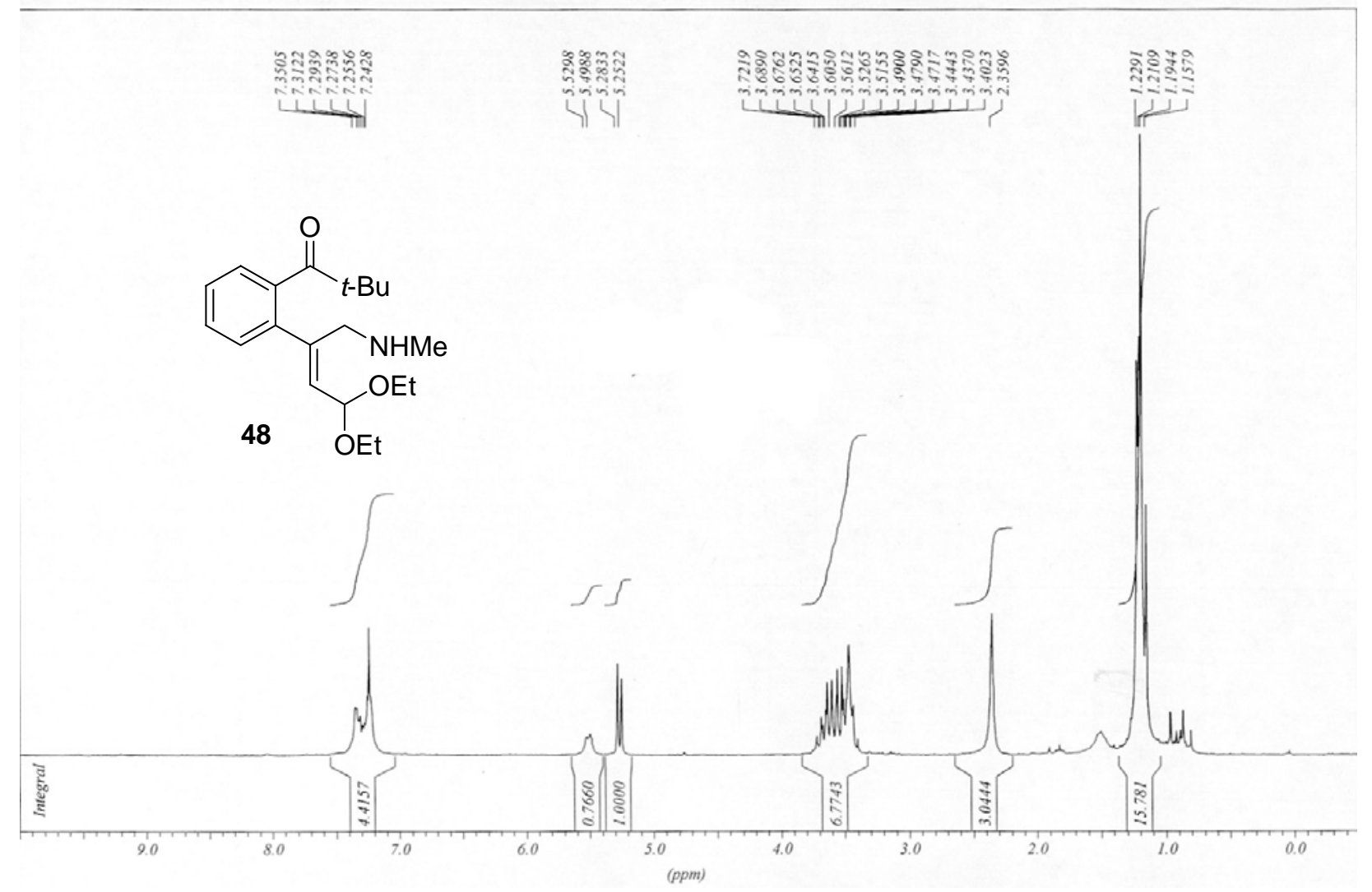

